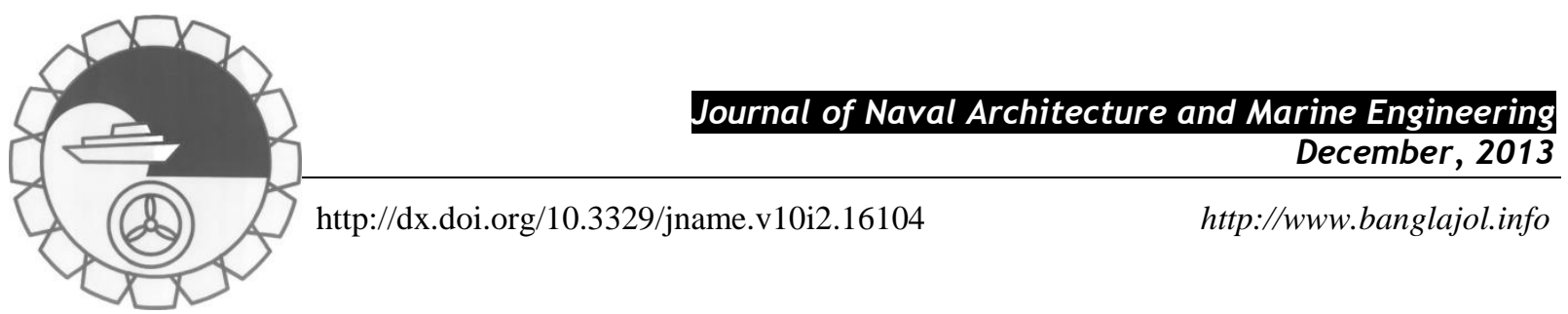

\title{
STUDY ON BOW WAVE BREAKING AROUND ULTRA LARGE BLOCK COEFFICIENT SHIP
}

\author{
Md. Ashim Ali ${ }^{1}$, Kazuo Suzuki ${ }^{2}$ and Sou Miyauchi ${ }^{3}$ \\ ${ }^{1}$ Department of Naval Architecture \& Marine Engineering, Bangladesh University of Engineering \& Technology, Dhaka- \\ 1000, Bangladesh. Email: ashim.name@gmail.com \\ ${ }^{2}$ Faculty of Engineering, Yokohama National University, Japan \\ ${ }^{3}$ Undergraduate School of Engineering, Yokohama National University, Japan
}

\begin{abstract}
:
Due to the increase of maritime transportation volume day by day it is necessary to design a ship's hull having a large carrying capacity with low resistance. In case of slow-moving ships, usually wave breaking occurs in front of a bow. A considerable portion of resistance occurs due to the energy dissipation of such as wave breaking in case of Ultra Large Block coefficient Ship (ULBS) suggested by the authors. The key objective of this research work is to investigate the relationship between bow wave breaking and free surface disturbance function that may be used as a parameter for numerical prediction of bow wave breaking. In this regard, the experiments and numerical calculations have been carried out for six models of ULBS. From the results, it can be concluded that the wave breaking area in front of bow increases with the increase of the surface integral of the square of free surface disturbance function, Froude number and block coefficient.
\end{abstract}

Keywords: Wave breaking, free surface disturbance (FSD) function, Rankine source method, ultra large block coefficient ship (ULBS).

\section{Introduction}

Strategy in the world economy has been changed significantly, as the world's business is moving towards more globalization than ever before. As a result, it becomes indispensable to improve the maritime transportation efficiency with a higher carrying capacity. One of the possible ways to improve the transportation efficiency is to increase the power efficiency for large ocean-going vessels. Improved power efficiency demands a ship hull form should be optimized having a large block coefficient from the hydrodynamic point of view, i.e., with low wave making resistance as well as wave breaking resistance.

At a low speed, wave breaking resistance is the most important component of wave resistance, which occurs in front of a bow in case of large ocean-going vessels. Ship types like oil carriers, bulk carriers having a full hull form, produce short waves with unstable crests in the bow region at a low speed. With the decrease of a ship draft, those short waves gradually transformed into breaking waves. From wave and wake measurements of tanker models, Baba (1969) found that the resistance component due to wave breaking in front of a bow occupies a considerable portion of the total ship resistance in ballast loading condition.

From the hydrodynamic point of view, the wave resistance of a body near the free surface can be split into two components: the former related to the waves radiated far behind the body, the latter associated with the wave energy dissipated by wave breaking. To understand the wave breaking phenomena in front of a bow for full hull form, Baba (1969) showed that the increase of wave breaking resistance is due to the expenditure of energy in generating turbulence due to breakdown of waves at the bow of ships. Baba (1975) also showed that the effective horse power due to wave breaking is about $25 \%$ of total effective horse power at design speed (19 knots) for the model with normal bow, while for the model with protruded bow, this component is reduced to $10 \%$ of total effective horse power.

Baba $(1975,1976)$ showed from analytical calculations of semi-submerged ellipsoid that steeper waves give a higher peak value of Free Surface Disturbance (FSD) function. It is considered that the wave breaking phenomena will be suppressed by reducing the values of FSD function in front of a bow. Protruding bow works in cancelling FSD function values induced by the main body in front of a bow, i.e. the protruding bow is

1813-8235 (Print), 2070-8998 (Online) @ 2013 ANAME Publication. All rights reserved. $\quad$ Received on: August, 2013 
effective in reducing the steepness of local bow wave. The objective of present study is to correlate between wave breaking and FSD function. Compare to slow-ship method, the FSD function can be used as a key parameter for prediction of the bow wave breaking because of its' capability to calculate the slope and velocity of wave at a point on the free surface. FSD function is calculated by using Hess \& Smith method according to Baba's low-speed theory. It is mentioned here that both Baba's theory and Rankine source method is based on low speed assumption, and their basic double model flow can be obtained by using Hess \& Smith method. In the present study, wave elevations and wave making resistance coefficients are obtained by using Rankine source method. The flow diagram of present numerical calculation is presented in Fig. 1.

In the present study, to understand the wave breaking phenomena, i.e. wave breaking area on the free surface in front of bow, experiments have been carried out for six ULBS models of full hull form $\left(C_{b} \gtrsim 0.95\right)$.

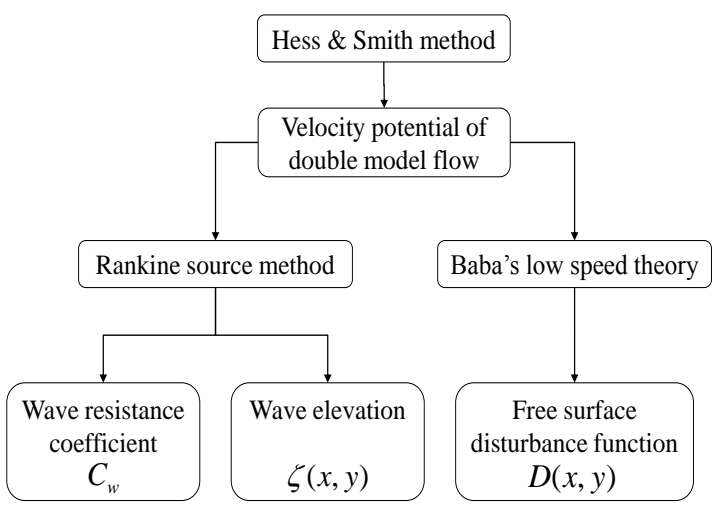

Fig. 1: Flow chart of present numerical calculation

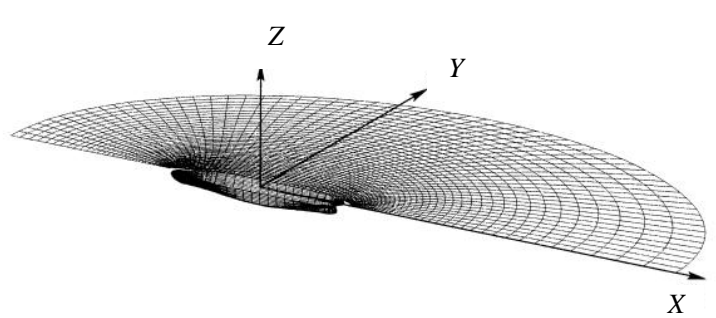

Fig. 2: Coordinate system and source panel arrangement

\section{Baba's Low Speed Theory and Free Surface Disturbance (FSD) Function}

In the following derivation of the free surface disturbance (FSD) function is cited from Baba (1976). Taking the rectangular coordinate system fixed on the body with the origin on a still water plane, the $x$-axis is set along the direction of the uniform flow $U$ and $z$-axis directing upwards as shown in Fig. 2. Assuming the ship is floating on an inviscid, irrotational, incompressible fluid, the velocity potential for free surface problem is expressed as a sum of two parts:

$$
\phi(x, y, z)=\phi_{0}(x, y, z)+\phi_{1}(x, y, z)
$$

where, $\phi_{0}(x, y, z)$ is the potential for the rigid-wall problem and $\phi_{1}(x, y, z)$ is an additional potential to $\phi_{0}(x, y, z)$ so that the sum satisfies the free surface conditions.

According to Ogilvie (1968), the wave height is assumed as the sum of two parts, i.e.

$$
H(x, y)=\zeta_{0}(x, y)+\zeta_{1}(x, y)
$$

where, $\zeta_{0}(x, y)$ is the wave height due to double body potential, i.e.

$$
\zeta_{0}(x, y)=\frac{1}{2 g}\left[U^{2}-\phi_{0 x}^{2}(x, y, 0)-\phi_{0 y}^{2}(x, y, 0)\right]
$$

Since $\phi_{0 z}(x, y, 0)=0, \zeta_{1}(x, y)$ is a superposed wave on $\zeta_{0}(x, y)$.

The boundary value problem for the present study is written as follows:

$$
\begin{aligned}
& \phi_{x x}+\phi_{y y}+\phi_{z z}=0 \\
& g H(x, y)+\frac{1}{2}\left[\phi_{x}^{2}+\phi_{y}^{2}+\phi_{z}^{2}\right]=\frac{1}{2} U^{2}, \text { on } z=H(x, y)
\end{aligned}
$$


$H_{x} \phi_{x}+H_{y} \phi_{y}-\phi_{z}=0$, on $z=H(x, z)$

$\phi_{n}=0, n$ is a normal vector on the body surface, and

$$
\phi-U x=\left\{\begin{array}{rr}
O\left(1 / \sqrt{x^{2}+y^{2}}\right) & \text { for } x>0 \\
\text { as } x^{2}+y^{2} \rightarrow \infty \\
o\left(1 / \sqrt{x^{2}+y^{2}}\right) & \text { for } x<0
\end{array}\right.
$$

By the substitution of Equations (1) and (2) into Equations (5) and (6), the free surface conditions are written as:

$$
\begin{aligned}
& \frac{1}{2}\left[U^{2}-\phi_{0 x}^{2}(x, y, 0)-\phi_{0 y}^{2}(x, y, 0)\right]+g \zeta_{1}(x, y)+\frac{1}{2}\left[\begin{array}{r}
\phi_{0 x}^{2}(x, y, z)+\phi_{1 x}^{2}(x, y, z)+2 \phi_{0 x}(x, y, z) \phi_{1 x}(x, y, z) \\
+\phi_{0 y}^{2}(x, y, z)+\phi_{1 y}^{2}(x, y, z)+2 \phi_{0 y}(x, y, z) \phi_{1 y}(x, y, z) \\
+\phi_{0 z}^{2}(x, y, z)+\phi_{1 z}^{2}(x, y, z)+2 \phi_{0 z}(x, y, z) \phi_{1 z}(x, y, z)
\end{array}\right]=\frac{1}{2} U^{2} \\
& {\left[\zeta_{0 x}(x, y)+\zeta_{1 x}(x, y)\right]\left[\phi_{0 x}(x, y, z)+\phi_{1 x}(x, y, z)\right]+} \\
& {\left[\zeta_{0 y}(x, y)+\zeta_{1 y}(x, y)\right]\left[\phi_{0 y}(x, y, z)+\phi_{1 y}(x, y, z)\right]-\phi_{0 z}(x, y, z)-\phi_{1 z}(x, y, z)=0}
\end{aligned}
$$

Based on Ogilvie's [8] assumptions,
(a) $\phi_{0}(x, y, z)=O(U)$,
(b) $\zeta_{0}(x, y, z)=O\left(U^{2}\right)$,
(c) $\frac{\partial}{\partial x}, \frac{\partial}{\partial x}, \frac{\partial}{\partial x}=O(1)$ when operating on $\phi_{0}(x, y, z)$ or $\zeta_{0}(x, y)$,
(d) $\phi_{1}(x, y, z)=O\left(U^{5}\right)$
(e) $\zeta_{1}(x, y, z)=O\left(U^{4}\right)$
(f) $\frac{\partial}{\partial x}, \frac{\partial}{\partial x}, \frac{\partial}{\partial x}=O\left(U^{-2}\right)$ when operating on $\phi_{1}(x, y, z)$ or $\zeta_{1}(x, y)$

The Taylor expansions at $z=0$ are derived for $\phi_{0 x}(x, y, z), \phi_{0 y}(x, y, z)$ and $\phi_{0 z}(x, y, z)$ are as follows:

$$
\begin{aligned}
& \phi_{0 x}(x, y, z)=\phi_{0 x}(x, y, 0)+\left(\zeta_{0}+\zeta_{1}\right) \phi_{0 x z}(x, y, 0)+\frac{1}{2}\left(\zeta_{0}+\zeta_{1}\right)^{2} \phi_{0 x z z}(x, y, 0)+\ldots \\
& =\phi_{0 x}(x, y, 0)+\frac{1}{2}\left(\zeta_{0}+\zeta_{1}\right)^{2} \phi_{0 x z z}(x, y, 0)+ \\
& {[U] \quad\left[U^{5}\right]} \\
& \phi_{0 y}(x, y, z)=\phi_{0 y}(x, y, 0)+\frac{1}{2}\left(\zeta_{0}+\zeta_{1}\right)^{2} \phi_{0 y z}(x, y, 0)+\ldots \ldots \\
& {[U] \quad\left[U^{5}\right]} \\
& \phi_{0 z}(x, y, z)=\zeta_{0}(x, y) \phi_{0 z z}(x, y, 0)+\zeta_{1}(x, y) \phi_{0 z z}(x, y, 0) \\
& {\left[U^{3}\right] \quad\left[U^{5}\right]}
\end{aligned}
$$

Where the following relations are used,

$$
\phi_{0 z}(x, y, 0)=0, \phi_{0 x z}(x, y, 0)=0, \phi_{0 y z}(x, y, 0)=0 \text {. }
$$

On the other hand, the Taylor expansions at $z=\zeta_{0}(x, y)$ are derived for $\phi_{1 x}(x, y, z), \phi_{1 y}(x, y, z)$ and $\phi_{1 z}(x, y, z)$ : 


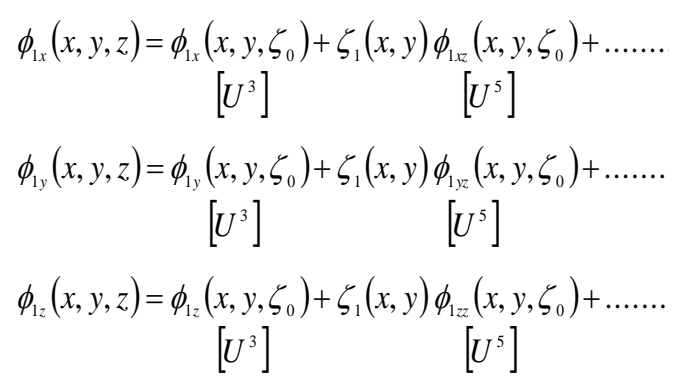

By substituting those expansions into Equations (8) and (9), and taking the lowest order terms, we have

$$
\begin{aligned}
& g \zeta_{1}(x, y)+\phi_{0 x}(x, y, 0) \phi_{1 x}\left(x, y, \zeta_{0}\right)+\phi_{0 y}(x, y, 0) \phi_{1 y}\left(x, y, \zeta_{0}\right)=0 \\
& -\phi_{1 z}\left(x, y, \zeta_{0}\right)+\zeta_{1 x}(x, y) \phi_{0 x}(x, y, 0)+\zeta_{1 y}(x, y) \phi_{0 y}(x, y, 0)+\zeta_{0 x}(x, y) \phi_{0 x}(x, y, 0)+\zeta_{0 y}(x, y) \phi_{0 y}(x, y, 0)-\zeta_{0}(x, y) \phi_{0 z z}(x, y, 0)=0
\end{aligned}
$$

By the use of the relation $\phi_{0 z z}=-\phi_{0 x x}-\phi_{0 y y}$, the Equation (17) can be written:

$$
\phi_{1 z}\left(x, y, \zeta_{0}\right)-\zeta_{1 x}(x, y) \phi_{0 x}(x, y, 0)-\zeta_{1 y}(x, y) \phi_{0 y}(x, y, 0)=\frac{\partial}{\partial x}\left[\zeta_{0}(x, y) \phi_{0 x}(x, y, 0)\right]+\frac{\partial}{\partial y}\left[\zeta_{0}(x, y) \phi_{0 y}(x, y, 0)\right]
$$

By neglecting the terms of $O\left(U^{4}\right)$ and substituting $\zeta_{x}(x, y)$ and $\zeta_{y}(x, y)$ into Equation (9), we finally have

$\frac{1}{g}\left[\phi_{0 x}(x, y, 0) \frac{\partial}{\partial x}+\phi_{0 y}(x, y, 0) \frac{\partial}{\partial y}\right]^{2} \phi_{1}\left(x, y, \zeta_{0}\right)+\phi_{1 z}\left(x, y, \zeta_{0}\right)=D(x, y)$ on $z=\zeta_{0}(x, y)$

where, $D(x, y)=\frac{\partial}{\partial x}\left[\phi_{0 x}(x, y, 0) \zeta_{0}(x, y)\right]+\frac{\partial}{\partial y}\left[\phi_{0 y}(x, y, 0) \zeta_{0}(x, y)\right]$

is the Free Surface Disturbance (FSD) function.

\section{Rankine Source Method}

The origin of the coordinate system is taken at the center of the hull on the free surface, where $x$ - axis is considered positive in the direction of uniform fluid velocity $U, y$-axis in the direction of starboard and the $z$ axis in upward direction as shown in Fig. 2. In Rankine source method, the fluid is considered inviscid and irrotational. The total velocity potential on the free surface, $\phi$ is the sum of velocity potential due to double model flow, $\phi_{0}$ and the perturbed velocity potential representing the effect of free surface, $\phi_{1}$.

$$
\phi=\phi_{0}+\phi_{1}
$$

Here, the velocity potential, $\phi_{0}$ due to double model flow can be represented as follows with the source density, $\sigma_{0}$ for the flow distributed on the body surface, $S_{0}$.

$$
\phi_{0}(x, y, z)=U x-\iint_{S_{0}} \sigma_{0}\left(x^{\prime}, y^{\prime}, z^{\prime}\right) \frac{1}{r_{0}} d S
$$

where,

$$
r_{0}=\sqrt{\left(x-x^{\prime}\right)^{2}+\left(y-y^{\prime}\right)^{2}+\left(z-z^{\prime}\right)^{2}}
$$

In addition, the velocity potential, which represents the effect of free surface, $\phi_{1}$ on the undisturbed surface can be expressed by

$$
\phi_{1}(x, y, z)=-\iint_{S_{1}} \sigma_{1}\left(x^{\prime}, y^{\prime}\right) \frac{1}{r_{1}} d x^{\prime} d y^{\prime}-\iint_{S_{0}} \Delta \sigma_{0}\left(x^{\prime}, y^{\prime}, z^{\prime}\right) \frac{1}{r_{0}} d S
$$

where, 


$$
r_{1}=\sqrt{\left(x-x^{\prime}\right)^{2}+\left(y-y^{\prime}\right)^{2}+z^{2}}
$$

The boundary conditions for hull surface require that the normal velocity on the hull must be zero. If the outward normal, $n$ on the hull surface then the hull surface boundary conditions are,

$$
\frac{\partial \phi_{0}}{\partial n}=0, \frac{\partial \phi_{1}}{\partial n}=0 \quad \text { on } S_{0}
$$

Free surface boundary conditions are represented as follows with free surface conditions of Dawson (1977) (double model linearized free surface condition) is,

$$
\phi_{0 l}^{2} \phi_{1 l l}+2 \phi_{0 l} \phi_{0 l l} \phi_{1 l}+g \phi_{1 z}=-\phi_{0 l}^{2} \phi_{0 l l} \text { on } S_{l}
$$

Wave elevation and the pressure around the hull can be determined from Bernoulli's equation by neglecting the higher-order terms of $\phi_{0}$ and $\phi_{1}$. The equation can be expressed as follows,

$$
p-p_{\infty}=\frac{1}{2} \rho\left(U^{2}-\phi_{0 x}^{2}-\phi_{0 y}^{2}-\phi_{0 z}^{2}-2 \phi_{0 x} \phi_{1 x}-2 \phi_{0 y} \phi_{1 y}-2 \phi_{0 z} \phi_{1 z}\right)
$$

And the wave profile is given as follows,

$$
\zeta(x, y)=\frac{1}{2 g}\left(U^{2}-\phi_{0 x}^{2}-\phi_{0 y}^{2}-2 \phi_{0 x} \phi_{1 x}-2 \phi_{0 y} \phi_{1 y}\right)
$$

\section{Visualization of Breaking Waves around Bow of ULBS Models}

\subsection{Model Tests of ULBS}

A schematic plan of ULBS suggested in Yokohama National University is shown in Fig. 3. For the practical goal of ULBS, various new ideas should be introduced to reduce fluid resistance and to improve propulsive performance. In the present paper, as one of the investigations for ULBS, fundamental studies on bow wave breaking are discussed.

For the study of wave breaking phenomena and FSD function, six ULBS models of different block coefficients $\left(C_{b} \gtrsim 0.95\right)$ are considered for the experiments, which have been carried out by the authors. Table 1 shows the principal particulars of ULBS models; Table 2 represents the test cases.

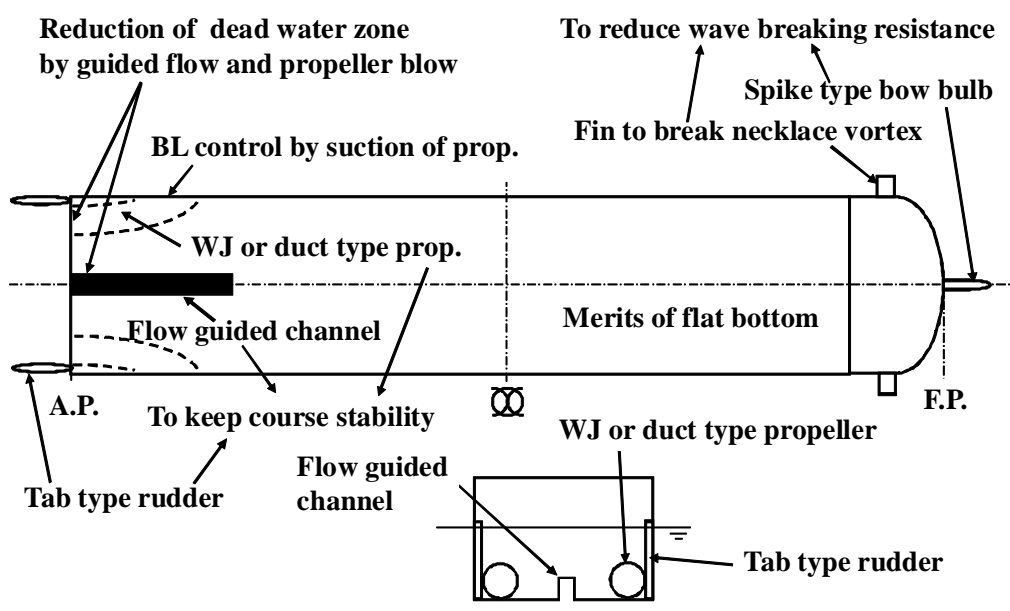

Fig. 3: Schematic plan of ULBS 
Table 1: Principal particulars of model ships

\begin{tabular}{|c|c|c|c|c|c|c|}
\hline & \multicolumn{2}{|c|}{ Type I } & \multicolumn{2}{|c|}{ Type II } & \multicolumn{2}{|c|}{ Type III } \\
\hline Length: $L[\mathrm{~m}]$ & \multicolumn{6}{|c|}{1.500} \\
\hline Breadth: $B[\mathrm{~m}]$ & \multicolumn{6}{|c|}{0.300} \\
\hline Depth: $D[\mathrm{~m}]$ & \multicolumn{6}{|c|}{0.250} \\
\hline Draft: $d[\mathrm{~m}]$ & 0.10 & 0.15 & 0.10 & 0.15 & 0.10 & 0.15 \\
\hline Block coefficient, $C_{b}$ & 0.948 & 0.949 & 0.960 & 0.962 & 0.973 & 0.974 \\
\hline Midship coefficient, $C_{m}$ & 0.995 & 0.997 & 0.995 & 0.997 & 0.995 & 0.996 \\
\hline Bilge radius, $R \quad[\mathrm{~m}]$ & \multicolumn{6}{|c|}{0.0187} \\
\hline
\end{tabular}

Table 2: Test cases for model ship

\begin{tabular}{|l|c|c|c|c|c|c|}
\hline Draft, $d[\mathrm{~m}]$ & \multicolumn{3}{|c|}{0.10} & \multicolumn{3}{c|}{0.15} \\
\hline$F_{n}$ & 0.103 & 0.129 & 0.155 & 0.126 & 0.158 & 0.190 \\
\hline$F_{n d}$ & 0.400 & 0.500 & 0.600 & 0.400 & 0.500 & 0.600 \\
\hline
\end{tabular}

\subsection{Formulations for Model Ship}

Formulations of ULBS models in this study are as follows. Symbols used in these formulations are given in Figs. 4 and 5.

The midship section coefficient,

$$
C_{m}=1+\frac{R^{2}(\pi-4)}{4 b d}
$$

Displacement of model,

$$
\nabla=L B d C_{m}+\frac{a B d C_{m}(\pi-4)}{4}
$$

Block coefficient of the model,

$$
C_{b}=C_{m}+a \frac{C_{m}(\pi-4)}{4 L}
$$

From Equation (32), the length of elliptic section for the model ship,

$$
a=\frac{4 L\left(C_{m}-C_{b}\right)}{C_{m}(4-\pi)}
$$

The maximum half breadth in the elliptic section of the hull,

$$
b_{i}=b \cdot \sqrt{1-\frac{1}{a^{2}}\left(x-\frac{L}{2}+a\right)^{2}}
$$

The half breadth at midship,

$$
y_{b}=b-R(1-\sin \theta)
$$

And the half breadth at the elliptic section,

$$
y_{b i}=\frac{b_{i}}{b} y_{b}
$$




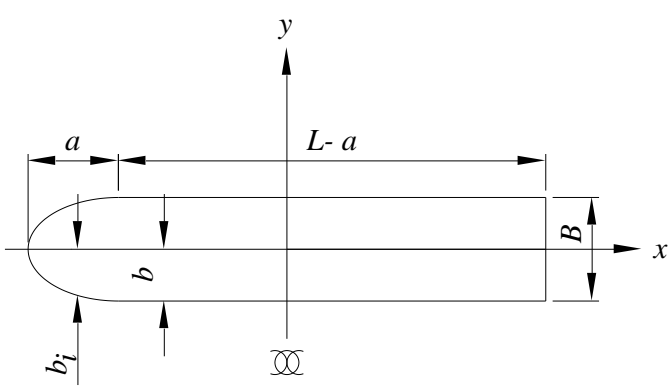

Fig. 4: Water plane of an ULBS model.

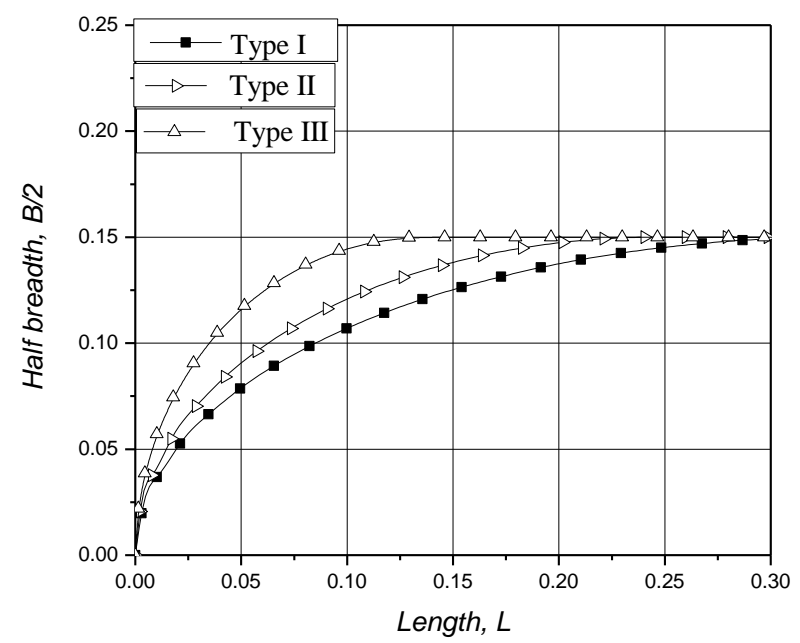

Fig. 6: Shape of bow of ULBS models.

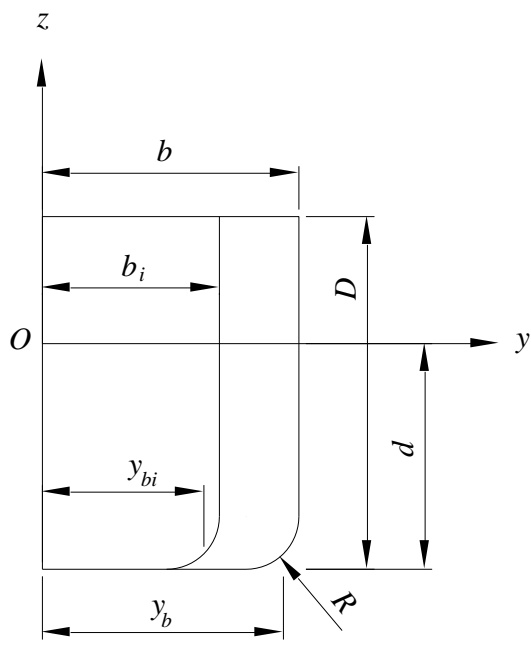

Fig. 5: Cross section of an ULBS model.

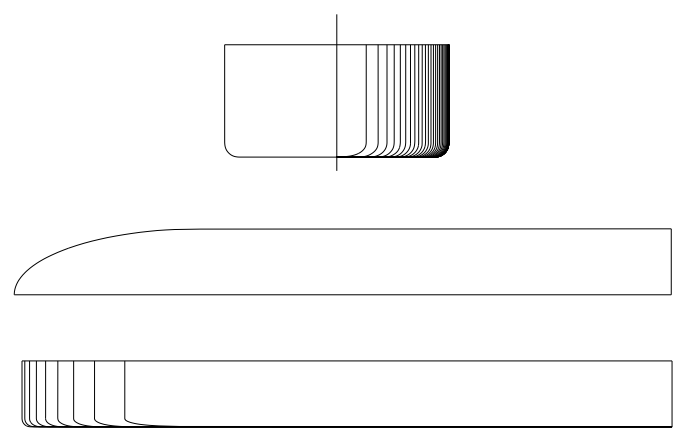

Fig. 7: Lines plan of an ULBS model.

The shape of bow and the lines plan of tested models of ULBS are shown in Figs. 6 and 7 respectively. For numerical calculation, the hull is defined by using the quadrilateral panels. The hull surface consists of 3402 panels and 3608 points. The free surface consists of 4800 quadrilateral panels having the elliptical boundary. For an example, panel distribution on hull and free surface for a ULBS model is shown in Fig. 8.

\subsection{Experimental Visualization Method}

Ship model is fixed on the free surface in the testing part of the circulating water channel during experiments. Usually capillary waves are observed in front of the model, as the surface tension effect becomes greater and greater relatively for small-scale free surface phenomena. In order to reduce the surface tension effect, a water solution of surface activator (surfactant) is sprayed on the free surface at upstream of the model. In experiments, the surface activator is very convenient to change the surface tension on the free surface. According to the experimental method suggested by Suzuki et al. (2008), the wave breaking area in front of bow, $S_{W B}$ is visualized by using a flat plate with longitudinal white and black stripes placed on the bottom of the circulating water channel. Electric lamps are used over the free surface for lighting. Therefore, wave patterns can be recorded easily by digital camera, which is placed above the free surface. With this experimental technique, it is possible to visualize the wave breaking clearly. Fig. 9 shows the wave breaking area of a ULBS model. The effects of surfactant are described in Appendix A1. 


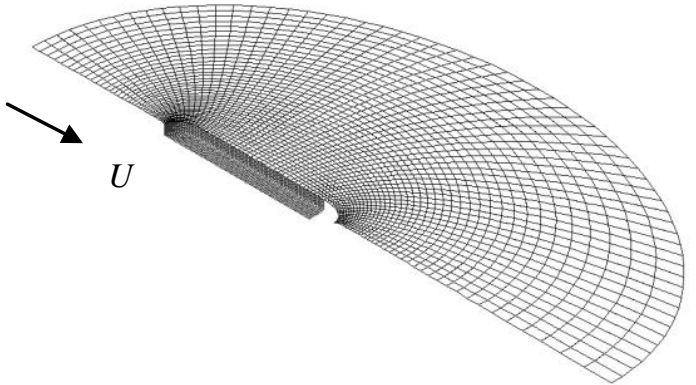

Fig. 8: Panels distribution on hull and free surface

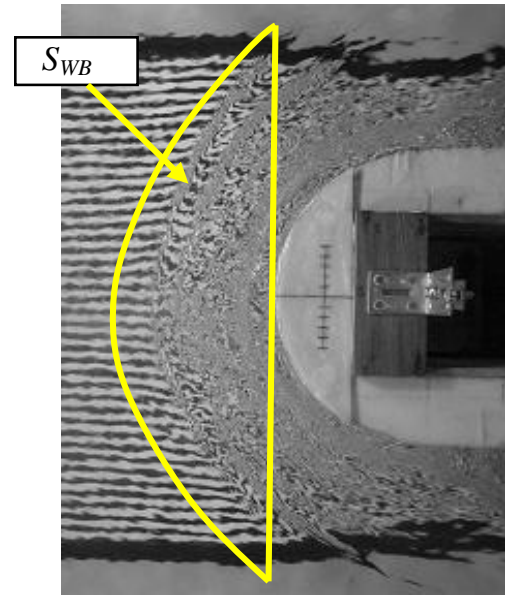

Fig. 9: Definition of wave breaking surface area, $S_{W B}$ in front of bow for model having $C_{b}=0.974$ at $F_{n d}=0.50$

\section{Results and Discussion}

Baba (1969) showed that wave breaking resistance components can be separated by using wake survey analysis behind a ship model. This is because of wake distributions, which are influenced by the head loss due to the wave breaking in front of the bow. However, wake survey cannot be easily applied for practical applications. Therefore, in the present study, the wave breaking area is used as the experimental parameter instead of the wave breaking resistance coefficient based on the wake survey analysis.

One-half of the computational domain is used for numerical treatment since the hull surface of ULBS is symmetrical about the $x z$-plane. The free surface panel distribution starts from $1.5 \mathrm{~L}$ upstream to $2.5 \mathrm{~L}$ downstream ( $L=$ ship length) having the elliptical boundary.

In present study, ship models with two different drafts are used. Froude number $\left(F_{n d}\right)$ is defined based on the draft of the model $(d)$ to normalize the effect of the draft in numerical calculation. Since wave making resistance is related the square of wave amplitude, the parameter $I_{\zeta^{2}}$ is introduced, For wave breaking, the parameter $I_{D^{2}}$, is introduced as the integral of the square of FSD function in front of the bow, since FSD function can be used as a measure of wave breaking inception according to Baba's consideration (Baba, 1975). In future works, both parameters are expected as the objective function in ULBS bow form optimization problems.

$$
\begin{aligned}
& F_{n d}=\frac{V}{\sqrt{g d}} \\
& I_{\varsigma^{2}}=\int_{0}^{\infty} \int_{-0.5 L}^{-\infty} \varsigma^{2}(x, y) / L^{2} d x d y \\
& I_{D^{2}}=\int_{0}^{\infty} \int_{-0.5 L}^{-\infty} D^{2}(x, y) / U^{2} d x d y
\end{aligned}
$$

To obtain the value of numerical parameter $I_{D^{2}}$, FSD function is calculated using the mathematical procedure described in Akima $(1978,1984)$ and Hess \& Smith (1964).

\subsection{Effect of Depth and Block Coefficient on Free Surface Wave and FSD function}

The wave heights are calculated using Rankine source method for the six ULBS models. The wave height increases with the increase of depth of the model as shown in Figs. 10(a) and 10(b). The wave height also increases with the increase of the block coefficient of ULBS models having the same drafts, which are shown in Figs. 10(b) and 10 (c). The increase in angle of entrance leads the increase of wave height. Figs. 11(a) to 11(c) show calculated FSD on the free surface using Baba's low speed theory. The FSD function also increases with the increase of draft and block coefficient of ULBS models. 


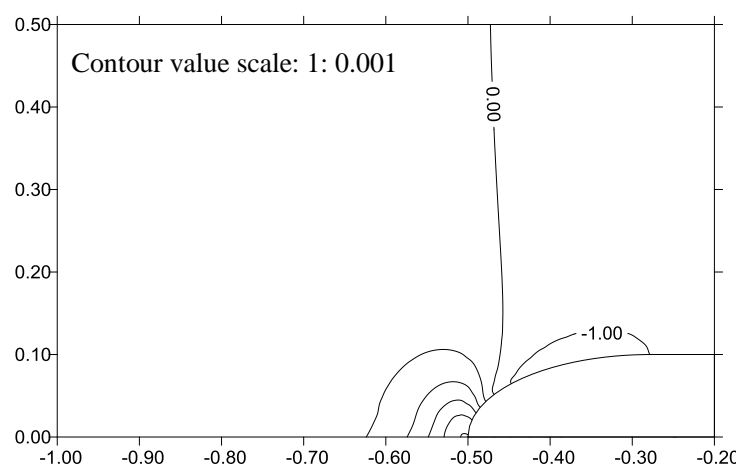

Fig. 10(a): Wave contour around the bow of ULBS having $C_{b}=0.948$ at $F_{n d}=0.40(d=0.10)$

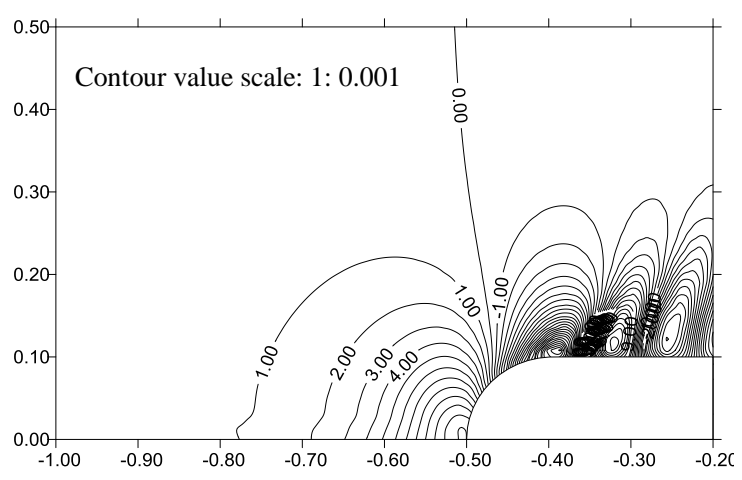

Fig. 10(c): Wave contour around the bow of ULBS having $C_{b}=0.974$ at $F_{n d}=0.50(d=0.15)$

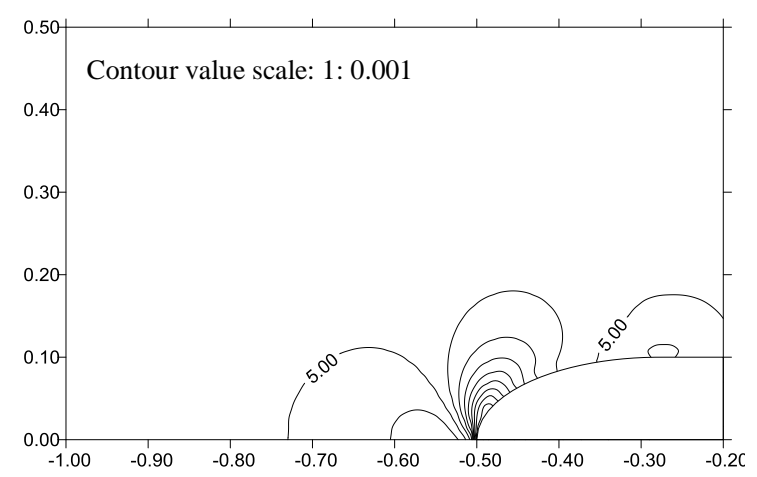

Fig. 11(b): Contour of FSD function around the bow of ULBS having $C_{b}=0.949$ at $F_{n d}=0.40$ $(d=0.15)$

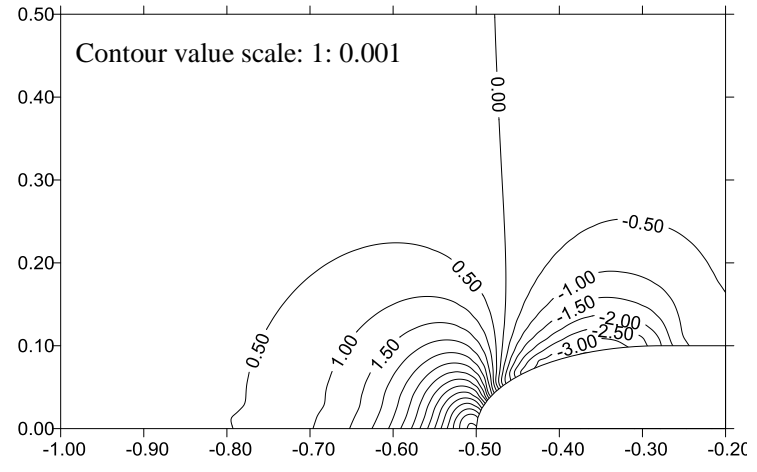

Fig. 10(b): Wave contour around the bow of ULBS having $C_{b}=0.949$ at $F_{n d}=0.40(d=0.15)$

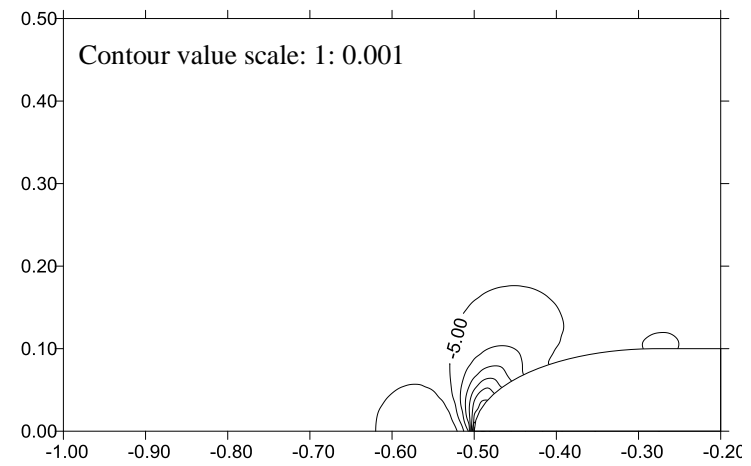

Fig. 11(a): Contour of FSD function around the bow of ULBS having $C_{b}=0.948$ at $F_{n d}=0.40$ $(d=0.10)$

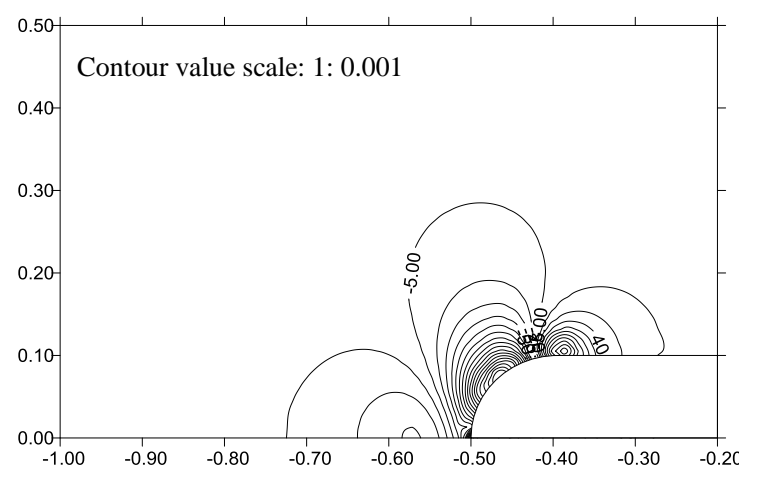

Fig. 11(c): Contour of FSD function around the bow of ULBS having $C_{b}=0.974$ at $F_{n d}=0.50$ $(d=0.15)$

\subsection{Effect of Block Coefficient on Wave Resistance Coefficient}

The effect of block coefficient, $C_{b}$ on wave resistance coefficient, $C_{w}$ is shown in Fig. 12. It is seen from Fig. 12 that at low speed block coefficient, $C_{b}$ has less effect on wave resistance coefficient, $C_{w}$ whereas at high speed, $C_{b}$ has significant influence on $C_{w}$. This may be due to the blunt bow form of ULBS models.

\subsection{Correlations Between Experimental and Numerical Parameters}

Fig. 13 shows the relation between $F_{n d}$ and $I_{\zeta^{2}}$. The value of $I_{\zeta^{2}}$ increases with the increase of $F_{n d}$. The increasing rate of change of $I_{\zeta^{2}}$ is significant at a higher draft if the other parameters remain constant. The relationship between $F_{n d}$ and $I_{D^{2}}$ is shown in Fig. 14. The rate of change in $I_{D^{2}}$ increases with the increase of 
$F_{n d}$ if the other parameters remain constant. If $d / B$ remains constant, $I_{D^{2}}$ increases with the increase of $I_{\zeta^{2}}$ as shown in Fig. 15. On the other hand, $I_{\zeta^{2}}$ increases with the increase of $d / B$.

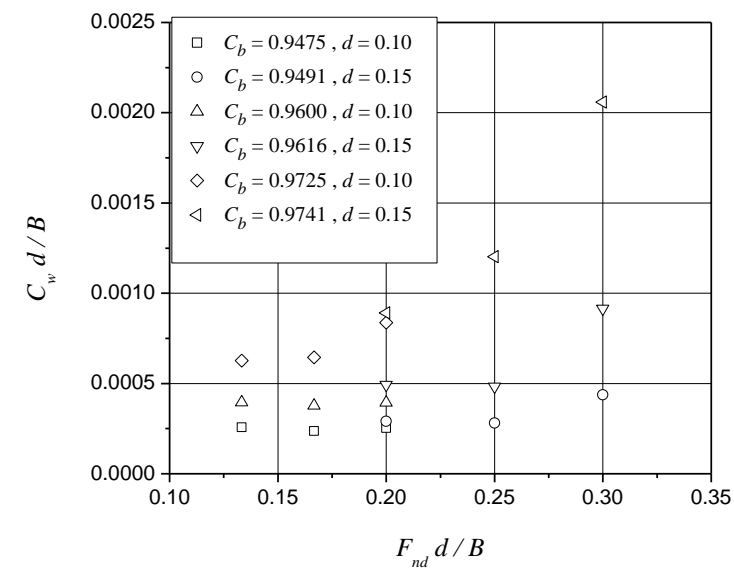

Fig. 12: Wave resistance coefficient, $C_{w}$

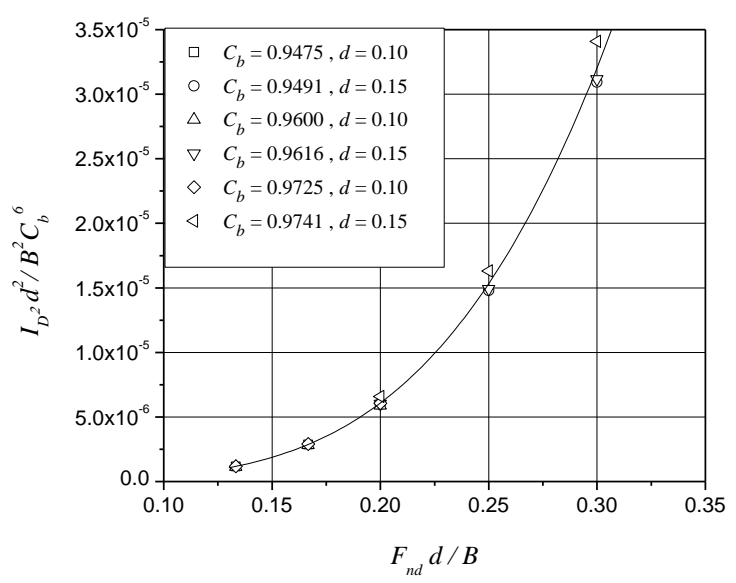

Fig. 14: Relationship between $F_{n d}$ and $I_{D^{2}}$

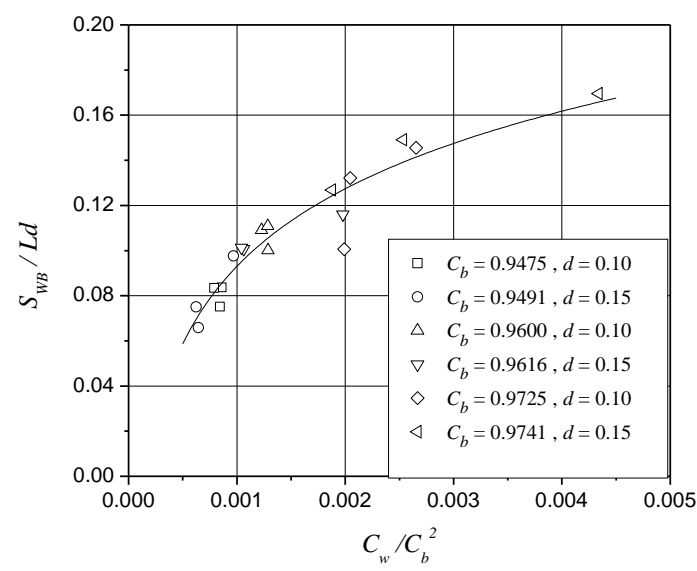

Fig. 16: Relationship between $C_{w}$ and $S_{W B}$

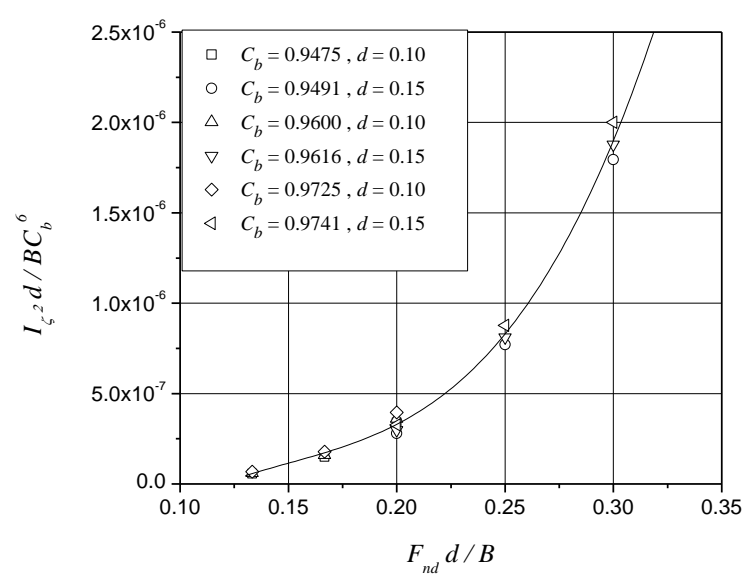

Fig. 13: Relationship between $F_{n d}$ and $I_{\zeta^{2}}$

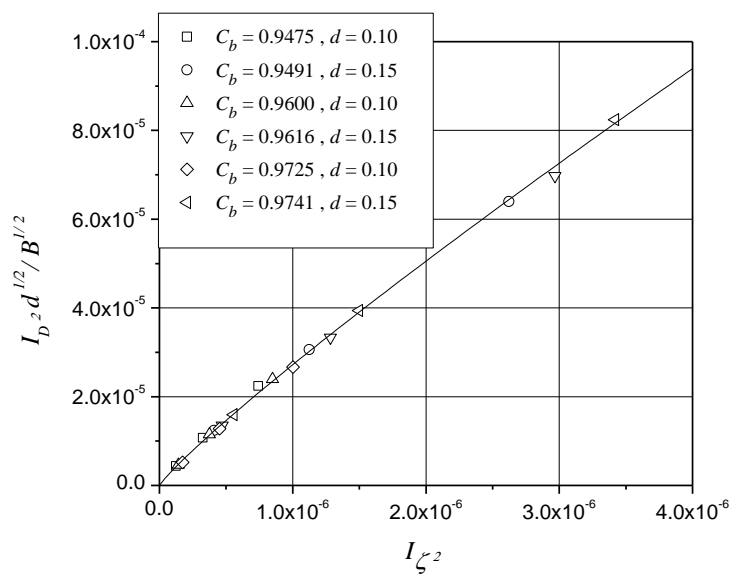

Fig. 15: Relationship between $I_{\zeta^{2}}$ and $I_{D^{2}}$

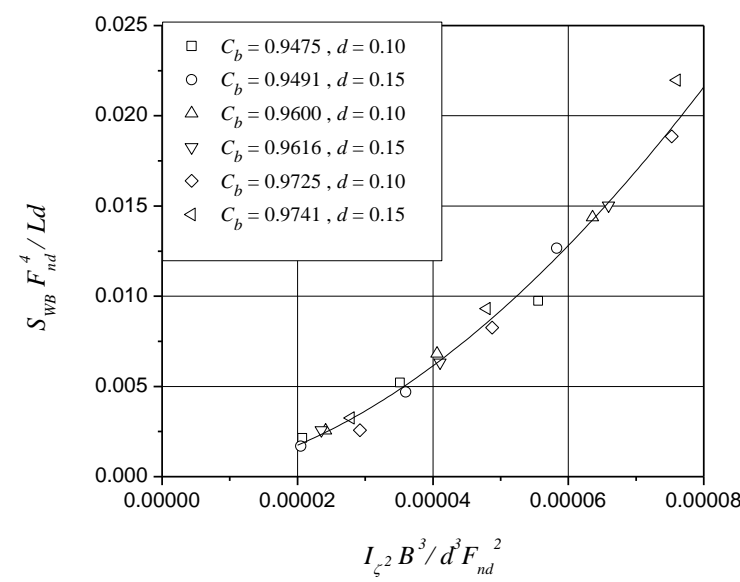

Fig. 17: Relationship between $I_{\zeta^{2}}$ and $S_{W B}$ 
In Fig. 16, the wave breaking surface area, $S_{W B}$ is plotted against wave resistance coefficient, $C_{w}$. From Fig. 16 , it is seen that for a low wave resistance coefficient, the wave breaking surface area increases remarkably with the increase of the wave resistance coefficient. However, at the higher range of the wave resistance coefficient, the rate of change in wave breaking surface area, $S_{W B}$ gradually decreases.

For six ULBS models having different block coefficients, the wave breaking surface area, $S_{W B}$ is plotted against $I_{\zeta^{2}}$ and shown in Fig. 17. From Fig. 17, it is noticed that with the increase of $I_{\zeta^{2}}$ and block coefficients, the wave breaking surface area in front of bow, $S_{W B}$ increases.

Fig. 18 shows the relation between wave breaking surface area, $S_{W B}$ and $I_{D^{2}}$ for the six ULBS models. From Fig. 18, it is observed that there is a strong relation between $S_{W B}$ and $I_{D^{2}}$, which is almost similar to the relation between $S_{W B}$ and $I_{\zeta^{2}}$. Keeping the other parameters constant, with the increase of $I_{D^{2}}$ and block coefficient, the rate of change of $S_{W B}$ increases.

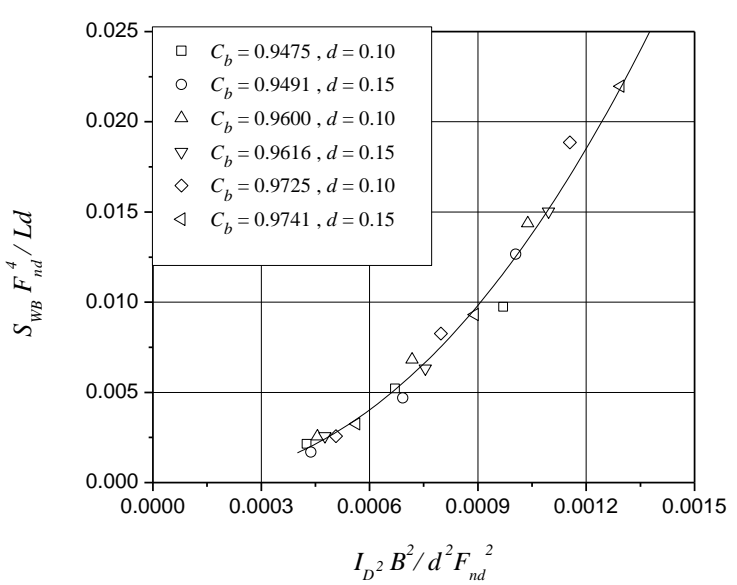

Fig. 18: Relationship between $I_{D^{2}}$ and $S_{W B}$

\section{Conclusions}

To measure the wave breaking area, $S_{W B}$ on the free surface in front of the bow, the experiments have been carried out for six ULBS models of full hull form $\left(C_{b} \gtrsim 0.95\right)$ by the authors. The numerical calculations are carried out using Rankine source method together with Hess \& Smith method to establish the relationship between wave breaking and FSD function. The following conclusions can be drawn based on the interpretation between experimental and numerical results;

1. With the increase of $F_{n d}$, the intensity of FSD function increases.

2. With the increase of $C_{w}$, the rate of the change in $S_{W B}$ gradually decreases.

3. With the increase of block coefficient, $I_{\zeta^{2}}$ and $I_{D^{2}}$, the rate of change in $S_{W B}$ increases.

Based on the present study, the FSD function can be used as a parameter to predict the wave breaking area and wave making resistance.

Current research survey indicates that the relationship established between wave breaking and FSD function for ULBS in the present study is the first of this kind. Since basic hull form without any appendages like bulbous bow, skeg or bilge keel is considered for present numerical calculation, the following future works need to be carried out:

1. Interpretation of FSD functions on the entire free surface.

2. Numerical calculation for hull with bulbous bow and other appendages.

3. Optimization of ULBS hull form. 


\section{Acknowledgement}

The first author acknowledges the Ministry of Education, Culture and Science of Japan (Monbukagakusho) for financial support during this research. The authors would also like to thank Mr. I. Okada (Yokohama National University), Dr. T. Kanai (Ship Research Center of Japan), Dr. Mir Tareque Ali and Dr. Md. Mashiur Rahaman (Bangladesh University of Engineering and Technology) for their useful discussion and suggestions.

\section{References}

1. Akima, H. (1978): A method of Bivariate Interpolation and Smooth Surface Fitting for Values Given at Irregularly Distributed Points, ACM Transactions on Mathematical Software, Volume 4, Number 2.

2. Akima, H. (1984): On Estimating Partial Derivatives for Bivariate Interpolation of Scattered Data, Rocky Mountain Journal of Mathematics, Volume 14, Number 1. http://dx.doi.org/10.1216/RMJ-1984-14-1-41.

3. Baba, E. (1969): Study on Separation of Resistance Components, Mitsubishi Technical Bulletin. No.59. http://dx.doi.org/10.2534/jjasnaoe1968.1969.9

4. Baba, E. (1975): Blunt Bow Forms and Wave Breaking, The First STAR Symposium on Ship Technology and Research. The Society of Naval Architects and Marine Engineers.

5. Baba, E. (1976): Wave Resistance of Ships in Low Speed, Mitsubishi Technical Bulletin, No.109.

6. Dawson, C.W. (1977): A Practical Computer Method for Solving Ship Wave Problem, 2nd Int. Conf. Numerical Ship Hydrodynamics. Berkeley.

7. Hess, A. J. and Smith, A.M.O, (1964): Calculation of Non-lifting Potential Flow about Arbitrary Threedimensional Bodies, Journal of Ship Research, pp 22-44.

8. Ogilvie, T. F. (1968): Wave Resistance: The Low Speed Limit, Univ. of Michigan, Naval Architecture and Marine Engineering, No. 002.

9. Suzuki, K., Ohkoshi, S., Ohno, K., Hirai, M., Akibayashi, H. and Yamamoto, S. (2008): Experimental Visualization of Kelvin Wave Pattern around Small-scale Ship Model in Circulating Water Channel, ISFV13$13^{\text {th }}$ International Symposium on Flow Visualization, France.

\section{Appendix A1}

The effects of surfactant are confirmed according to the reference [9], as the summarized experimental procedures are mentioned in section 4 of the present paper. For an example, surfactant of $2 \%$ water solution of cleanser "Woolite" containing 22.5\% surface activator is used as the spray material. In Fig. A1, measured surface tension forces of the water solution of "Woolite" are compared with that of pure water. As shown in Fig. A1, the surface tension force can be decreased remarkably by using the water solution of "Woolite", and $2 \%$ water solution can be judged as the most suitable condition from the viewpoints of surface tension force and spray easiness. Examples of visualized wave pattern around a small model are shown in Fig. A2. For decreasing the surface tension force by using the surfactant as shown in Fig. A2, ripples due to the surface tension effect can be almost disappeared. By using this experimental technique, clear visualizations of wave breaking area can also be expected for ULBS models.

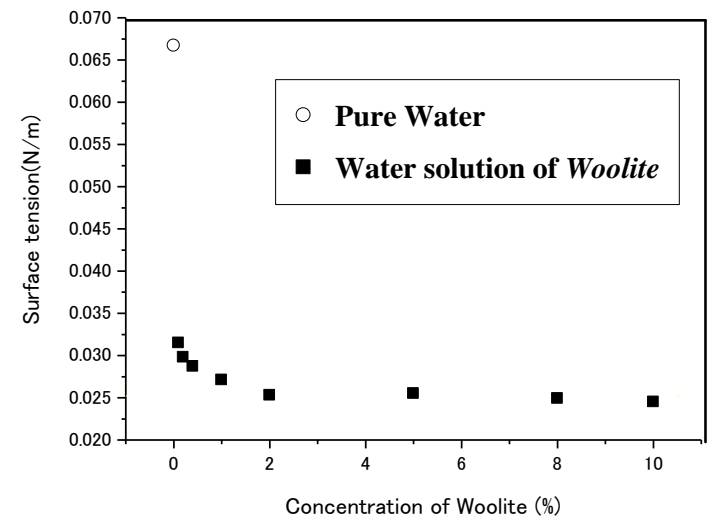

Fig. A1: Comparison of surface tension forces
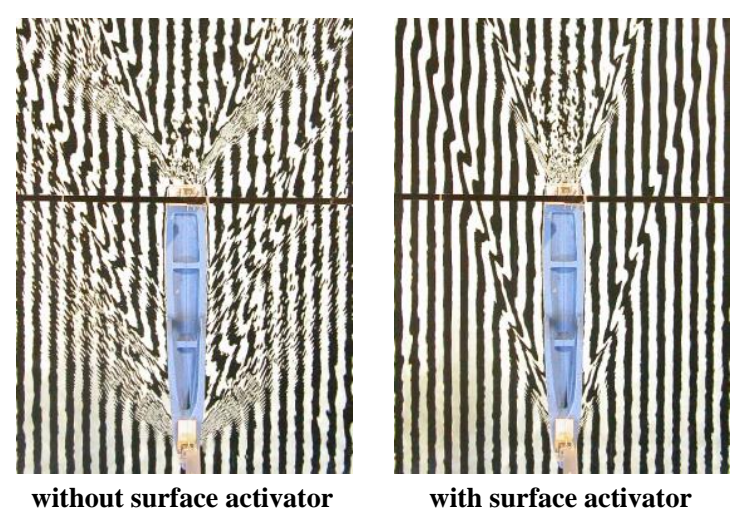

Fig. A2: Comparison of wave pattern around small model 